\section{Unpredictable Effects of Chemical Mixtures on Liver in Health and Disease}

\author{
Pinar Erkekoglu ${ }^{1 *}$ and Belma Kocer Giray ${ }^{1}$ \\ ${ }^{1}$ Hacettepe University, Faculty of Pharmacy, Turkey
}

Liver is the primary organ for the detoxification, as well as being the target of many of environmental chemicals. Today, we are all exposed to mixtures of different chemicals at a time because of industrialization and fast life style. Other than environmental chemicals, many people are subject to a mixture of drugs. For example, patients of complex diseases receive an average of six drugs at time when hospitalized. Home influenza treatment consists of analgesics, antihistaminics, and cough syrups. Yet, exposure to an antioxidant and chemical/drug together might be hepatotoxic, if the antioxidant is not applied at a proper dosage or if they affect the same enzymes, receptors, or pathways. Even drinking water may contain residues of pesticides, Polychlorinated Biphenyls (PCBs) along with heavy metals, which all possess hepatic toxicity potential. Exposure to different xenobiotics or administration of several drugs at a time may change the effect of each single component or may affect course of a disease. Although the basics of dose-response principle are rather well-accepted for mixtures, several toxicological challenges, including dose metrics and the non-quantified effects of toxicity modifying factors, complicate the interpretation of the obtained data and restrain the ability to interrelate the presence, nature, and extent of interactions between different components of a mixture.

From a public health perspective and as toxicologists, we can state that it is more difficult to predict the hepatotoxicity of chemical mixtures than a pure chemical, as each substance displays its own toxicity, and they may interact to produce additive, synergistic, or antagonistic effects. Lack of adequate scientific information about dose level influences and toxicokinetics/dynamics of several new environmental chemicals as well as species-specific variations contribute further interpretational limitations. Some of the mechanisms involved in alterations in toxicity have been studied and have been shown to be a consequence of changes in the toxicokinetics and/or toxicodynamics of one chemical by another. Toxicokinetic interactions between the components may affect the absorption, distribution, metabolism and/or excretion of a chemical and can have profound effects on dose-response relationships. Alterations at the toxicodynamic events might involve a competition between chemicals for binding to a target site, such as a receptor, or the susceptibility of target cells to the effects of another agent. This might also cover alterations in signal transduction pathways and cell cycle progress. Though some of these changes have been described for simple mixtures of two components, unraveling the effects of complex mixtures with up to hundreds of chemicals has not been attempted to an appreciable extent. Moreover, as the toxicity of chemicals are subject to change in a disease state, liver diseases as well as liver failure, or transplantation makes the situation more complex and hard to interpret with the available knowledge.

More complex are situations with more than one type of toxic entity, such as exposure to with both chemical and biological/ physical agents. For instance, people infected with hepatitis B (HBV) are at greater risk for developing aflatoxin B1 (AFB1) induced hepatocellular carcinoma and this interaction is widely studied by different researchers, who have been suggested a number of possible mechanisms for this interaction. Chronic HBV infection may induce the hepatic cytochrome P450s that metabolize AFB1 to its mutagenic metabolite, AFB1-8,9-epoxide. Moreover, reactive oxygen and nitrogen species resulting from chronic HBV infection cause hepatocyte necrosis and regeneration and raise the likelihood of the AFB1-induced p53 249ser and other mutations and the subsequent clonal expansion of cells containing these mutations. Although AFB1-DNA adducts are removed by nuclear excision repair, it is inhibited by HBV x protein, favoring the permanency of existing mutations. This protein also elevates the overall frequency of DNA mutations, including the p53 249ser mutation, and may contribute to uncontrolled cell cycling when p53 is non-functional [1-3].

In conclusion, we can state that hepatic toxicity of chemical and/ or biological mixtures may have serious effects on the functions of liver, which is the non-stopping machine for biotransformation as well as several very important physiological processes. This subject needs serious concern of researchers and medical doctors and new research should concentrate on this topic seriously. Developing novel statistical and mathematical strategies to interpret results can provide better models for predictive toxicology.

\section{References}

1. Kew MC (2003) Synergistic interaction between aflatoxin B1 and hepatitis B virus in hepatocarcinogenesis. Liver Int 23: 405-409.

2. Shirabe K, Toshima T, Taketomi A, Taguchi K, Yoshizumi T, et al. (2011) Hepatic aflatoxin B1-DNA adducts and TP53 mutations in patients with hepatocellular carcinoma despite low exposure to aflatoxin B1 in southern Japan. Liver Int 31: 1366-1372.

3. Gursoy-Yuzugullu O, Yuzugullu H, Yilmaz M, Ozturk M (2011) Aflatoxin genotoxicity is associated with a defective DNA damage response bypassing p53 activation. Liver Int 31: 561-571. 